\title{
Descoberta de carcinoma de células escamosas em lesão previamente diagnosticada como líquen plano oral: malignização ou erro de diagnóstico inicial?
}

Discovery of squamous cell carcinoma in a lesion previously diagnosed as oral lichen planus: malignant trasformation or wrong initial diagnosis?

Descubrimiento de carcinoma espinocelular en lesión previamente diagnosticada como líquen plano oral: malignización o error de diagnóstico inicial

Adriana Caroline LEITE 1

Nathalia Torres ASSAY 1

Fábio Auguto ITO2

Heliton Gustavo de LIMA ${ }^{2}$

Ademar TAKAHAMA JUNIOR ${ }^{2}$

${ }^{I}$ Graduação em Odontologia, Universidade Estadual de Londrina (UEL) 86057-970 Londrina - PR, Brasil

${ }^{2}$ Departamento de Medicina Oral e Odontologia Infantil, Curso de Odontologia, Universidade Estadual de Londrina (UEL) 86057-970 Londrina - PR, Brasil

\section{Resumo}

O Líquen Plano Oral (LPO) é uma doença crônica imunologicamente mediada com potencial de malignização incerto. O objetivo desse artigo é relatar um caso de Carcinoma de Células Escamosas (CCE) diagnosticado em paciente com diagnóstico prévio de LPO. Paciente do sexo feminino, 69 anos, foi atendida durante uma campanha de diagnóstico precoce de câncer de boca realizada na cidade de Londrina/PR, sendo detectada uma lesão eritroplásica em língua e encaminhada para uma melhor avaliação. Na anamnese a paciente relatou que há 2 anos atrás foi diagnosticada com LPO através de biópsia em língua, não sendo proposto nenhum tratamento. Ao exame físico, observamos uma mancha eritematosa em borda lateral de língua do lado esquerdo, não apresentando nenhuma outra lesão na cavidade oral. Com a hipótese diagnóstica de eritroplasia, foi requisitada a lâmina da biópsia anterior para análise, que revelou características microscópicas incompatíveis com o diagnóstico de LPO. Optamos então por realizar uma nova biópsia e a análise histopatológica revelou ilhas de células epiteliais malignas invadindo o tecido conjuntivo, estabelecendo o diagnóstico de CCE. A paciente foi encaminhada para tratamento, sendo realizada glossectomia parcial. Em acompanhamento de 6 meses a paciente encontra-se bem e sem sinais de recidiva. A transformação maligna do LPO ainda é discutida na literatura e muitos casos relatados podem se tratar de erro no diagnóstico inicial, como no caso apresentado. O erro de diagnóstico pode levar a morbidades importantes e comprometer o prognóstico do caso.

Descritores: Diagnóstico; Líquen Plano; Carcinoma de Células Escamosas.

\section{Abstract}

The Oral Lichen Planus (OLP) is a chronic inflammatory disease with uncertain malignant potential. The main purpose of this article is to report a case of a squamous cell carcinoma (SCC) in a patient with previous diagnosis of OLP. A 69-years-old female patient was examined during an oral cancer prevention campaign in Londrina/PR. At that occasion it was detected an erythroplakia in the tongue and the patient was referred for a better evaluation. The patient reported a previous biopsy and diagnosis of OLP in the tongue 2 years ago, without any treatment. At the physical examination, we observed an erythematous spot on the lateral border of the tongue on the left side, showing no other lesion in the oral cavity. With the diagnostic hypothesis of erythroplakia, the lamina of the previous biopsy was requested for analysis, which revealed microscopic characteristics incompatible with the diagnosis of OLP. We then chose to perform a new biopsy and the histopathological analysis revealed islands of malignant epithelial cells invading the connective tissue, establishing the diagnosis of SCC. The patient was referred for treatment and partial glossectomy was performed. At 6 months follow-up the patient is well and without signs of relapse. The malignant transformation of OLP is still discussed in the literature and many reported cases may be errors in the initial diagnosis, as in the case presented. The diagnostic error can lead to important morbidities and compromise the prognosis of the case.

Descriptors: Diagnosis; Lichen Planus; Carcinoma, Squamous Cell.

\section{Resumen}

El Líquen Plano Oral (LPO) es una enfermedad crónica inmunológicamente mediada con potencial de malignización incierta. El objetivo de este artículo es relatar un caso de Carcinoma de Células Escamosas (CCE) diagnosticado en paciente con diagnóstico previo de LPO. El paciente de sexo femenino, de 69 años, fue atendida durante una campaña de diagnóstico precoz de cáncer de boca realizada en la ciudad de Londrina / PR, siendo detectada una lesión eritroplásica en lengua y encaminada para una mejor evaluación. En la anamnesis la paciente relató que hace 2 años fue diagnosticada con LPO a través de biopsia me lengua, no siendo propuesto ningún tratamiento. En el examen físico, observamos una mancha eritematosa en borde lateral de lengua del lado izquierdo, no presentando ninguna otra lesión en la cavidad oral. Con la hipótesis diagnóstica de eritroplasia, se requirió la lámina de la biopsia anterior para análisis, que reveló características microscópicas incompatibles con el diagnóstico de LPO. Optamos entonces por realizar una nueva biopsia y el análisis histopatológico reveló islas de células epiteliales malignas invadiendo el tejido conectivo, estableciendo el diagnóstico de CCE. La paciente fue encaminada para tratamiento, siendo realizada glossectomía parcial. En seguimiento de 6 meses la paciente se encuentra bien y sin signos de recidiva. La transformación maligna del LPO todavía se discute en la literatura y muchos casos reportados pueden tratarse de error en el diagnóstico inicial, como en el caso presentado. El error de diagnóstico puede llevar a morbilidades importantes y comprometer el pronóstico del caso.

Descriptores: Diagnóstico; Liquen Plano; Carcinoma de Células Escamosas.

\section{INTRODUÇÃO}

O Líquen Plano Oral (LPO) é uma doença crônica imunologicamente mediada que pode ou não estar associada a lesões cutâneas. Os indivíduos acometidos por essa condição representam de 0,5 a $2 \%$ da população geral, sendo principalmente adultos de meia idade e raramente crianças. O LPO possui predileção pelo sexo feminino numa proporção de 3:2 em relação ao sexo masculino ${ }^{1-3}$.

A etiologia do LPO ainda não é conhecida, mas vários fatores têm sido relacionados ao seu desenvolvimento, principalmente o estresse e a hipersensibilidade. Sabe-se que o LPO é mediado por células citotóxicas que agridem os queratinócitos basilares, resultando em degeneração da camada basal do epitélio ${ }^{4,5}$.

O diagnóstico do LPO requer a associação das características clínicas e histopatológicas, podendo se assemelhar clinicamente com outras lesões. Os critérios clínicos para diagnóstico de LPO são a presença de lesões bilaterais; rendilhado com 
linhas brancas levemente elevadas (padrão reticular); formas erosivas, atróficas, bolhosas ou em placas são aceitas se houver lesões reticulares. Enquanto os critérios histopatológicos são os achados essenciais de infiltrado linfocitário disposto em banda subepitelial com apagamento da camada basal e ausência de displasia epitelial ${ }^{6}$.

Em 2003, Van der Meji e Van der Waal ${ }^{6}$ sugeriram mudanças nos critérios de diagnóstico de LPO definidos por Kramer et al. ${ }^{7}$ em 1978, a fim de distinguir o LPO da chamada lesão liquenoide, entidade que provavelmente apresentaria um maior potencial de malignização.

O potencial de malignização do LPO é bastante discutido, e apesar dos inúmeros estudos em torno do assunto, não há um consenso. Paralelamente aos casos de transformação maligna do LPO descritos na literatura, alguns estudos afastam a possibilidade de haver uma natureza pré-maligna desta lesão, sugerindo ainda que muitos dos casos que progrediram para um carcinoma podem ser consequência de erro do diagnóstico inicial ${ }^{1,6,8,9}$.

\section{CASO CLÍNICO}

Paciente do sexo feminino, de 69 anos, foi atendida durante uma campanha de diagnóstico precoce de câncer bucal realizada em um evento de saúde na cidade de Londrina-PR. Na ocasião foi detectada uma lesão eritroplásica em língua sendo então encaminhada para o Ambulatório de Estomatologia da Clínica Odontológica da UEL para uma avaliação mais detalhada. Durante a anamnese, a paciente relatou que há cerca de 2 anos atrás procurou vários profissionais, dentre eles dentista e médico cirurgião de cabeça e pescoço, sendo realizada uma biópsia com diagnóstico de Líquen Plano Oral, não sendo proposto nenhum tratamento. Ao exame físico, observamos uma área erosiva eritematosa, dolorosa, com limites indefinidos, localizada em borda lateral de língua do lado esquerdo (Figura 1) e não apresentando nenhuma outra lesão na cavidade oral. Com a hipótese diagnóstica principal de eritroplasia e com o quadro clinico incompatível com LPO, foi sugerida a realização de uma nova biópsia, porém, a paciente se mostrou resistente. Com isso, foi requisitada a lâmina da biópsia anterior para revisão. A análise da lâmina revelou um fragmento parcialmente ulcerado de mucosa, com intenso infiltrado inflamatório linfoplasmocitário difuso, incompatível com o diagnóstico de LPO (Figura 2). Desta forma, mais uma vez propomos à paciente a repetição da biópsia, que desta vez concordou em realizar. Nessa segunda biópsia observamos microscopicamente ilhas de células epiteliais malignas invadindo $o$ tecido conjuntivo (Figura 3), estabelecendo o diagnóstico de Carcinoma de Células Escamosas. A paciente foi encaminhada ao Hospital do Câncer de Londrina para tratamento, que consistiu em glossectomia parcial. Em acompanhamento de 12 meses a paciente encontra-se bem e sem sinais de recidiva. (Figura 4)

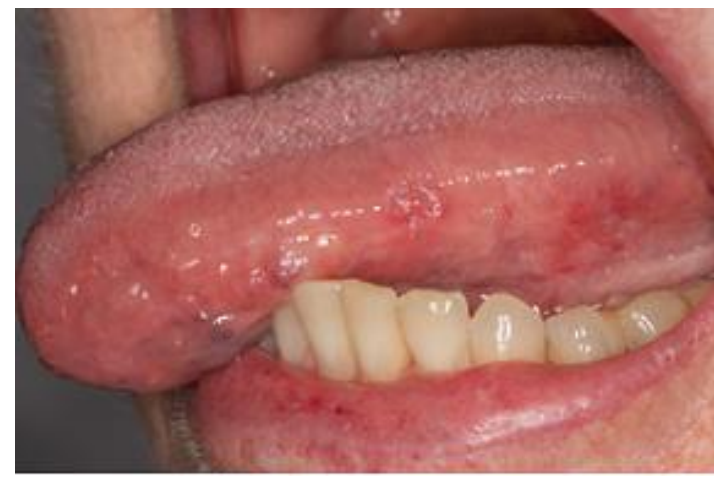

Figura 1: Área erosiva eritematosa, com limites difusos, localizada em borda lateral de língua do lado esquerdo.

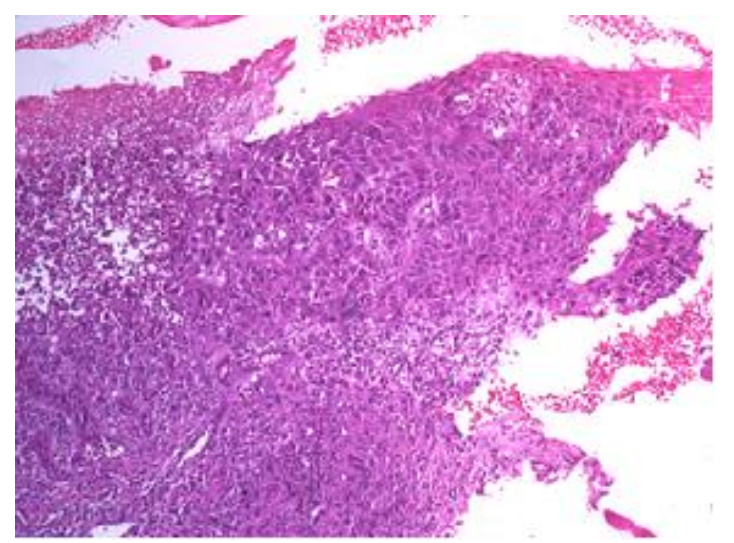

Figura 2: Fragmento de mucosa parcialmente ulcerado, com intenso infiltrado inflamatório linfoplasmocitário difuso. (HE, x200)

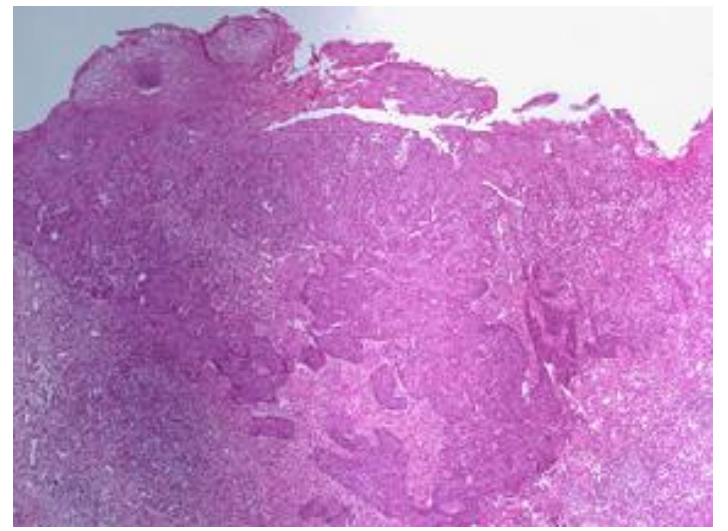

Figura 3: Fragmento de mucosa apresentando Ilhas de células epiteliais malignas invadindo o tecido conjuntivo. (HE, x100)

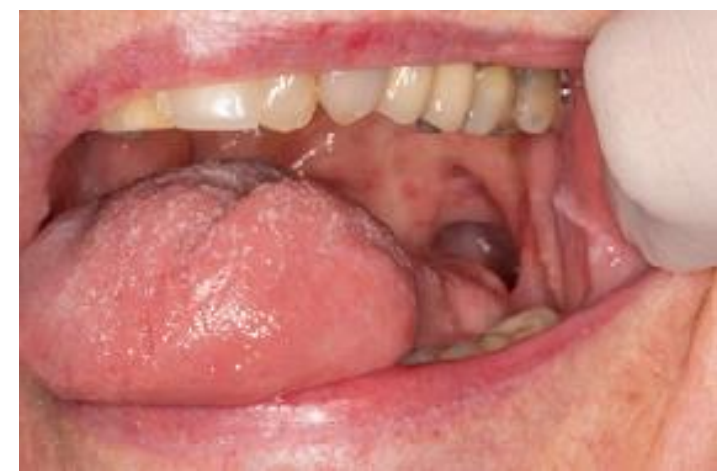

Figura 4: Aspecto clínico após 1 ano de acompanhamento. 


\section{DISCUSSÃO}

O líquen plano é uma desordem imunológica de acometimento mucocutâneo, que também pode surgir em pele e mucosa isoladamente. Quando acomete a mucosa oral é chamada líquen plano oral (LPO), que por sua vez, pode apresentar algumas variações clínicas e semelhanças com outras entidades patológicas ${ }^{10-12}$. Por isso, o seu diagnóstico deve ser feito sempre com a associação das características clínicas e microscópicas prédeterminadas. Van der Meji e Van der $\mathrm{Waal}^{6}$, em 2003, sugeriram mudança nos critérios da OMS de 1978 que possibilitou a diferenciação do LPO das lesões liquenoides, que representam lesões clinicamente e microscopicamente semelhantes ao LPO, mas que não preenchem todos os critérios estabelecidos para o seu diagnóstico. Uma das características microscópicas que podem ser encontradas nas lesões liquenoides é a displasia epitelial, o que pode explicar o possivel risco aumentado para transformação maligna dessas lesões. Van der Meji e van der Waal sugeriram ainda que essa diferenciação poderia afastar o potencial de malignização do LPO, indicando que provavelmente muitos casos de lesões liquenoides foram erroneamente diagnosticados como LPO.

No caso relatado houve provavelmente um erro de diagnóstico inicial, pois as características histopatológicas apresentadas na biópsia inicial eram incompatíveis com o LPO. Além disso, as características clínicas encontradas na nossa avaliação também não se apresentavam em concordância com os critérios clínicos do LPO.

A transformação maligna de casos antes diagnosticados como LPO é, inegavelmente, presente na literatura. A taxa de transformação maligna do LPO tem sido relatada por vários estudos entre $0,07 \%$ e $5,8 \%$, ao mesmo tempo em que outros autores ${ }^{13-16}$ apontam para nenhum potencial de malignização. Uma recente revisão sistemática de Giuliani et al. ${ }^{1}$, avaliou 43 publicações incluindo 6559 pacientes, e mostrou um potencial de malignização do LPO de $1,40 \%$, taxa maior daquela encontrada por Fitzpatrick et al. ${ }^{17}$ Aghbari etal. ${ }^{8}$, Bermejo-Fenoll et al. ${ }^{18}$ e Van der Meij et al. ${ }^{14}$, que concluíram o potencial de malignização de $1,09 \%, 1,1 \%, 0,91 \%$ e $0 \%$, respectivamente. Apesar disso, Giuliani et al. ${ }^{1}$ sugerem que para avaliar a verdadeira taxa de transformação maligna do LPO é necessário que o diagnóstico diferencial de LPO e lesão liquenoide seja feito a partir dos critérios da OMS modificados por Van der Meji e Van der Waal em 20036, reforçando que estudos futuros diagnostiquem o LPO e a lesão liquenóide seguindo esses ou novos critérios. Consideram ainda que os aspectos que poderiam influenciar significantemente o diagnóstico, consequentemente, influenciariam na metodologia da revisão, e que as mudanças dos critérios de 1978 até até agora, poderiam modificar consideravelmente a taxa de malignização final.

Alguns autores relacionam a inflamação crônica presente no LPO com o potencial de transformação maligna, indicando que aumento do estresse oxidativo em locais inflamados pela liberação de radicais livres, e a liberação de um grande número de fatores de crescimento e mediadores de inflamação, podem predispor o aparecimento e estabilização de mutações ${ }^{19,}{ }^{20}$. Por outro lado, há evidencias científicas de que o LPO apresenta perda de heterozigosidade e instabilidade de microssatélites mais semelhantes a lesões benignas da mucosa oral, do que em displasias e lesões malignas $^{21}$. Portanto, cientificamente não há unanimidade em relação ao potencial de malignização do LPO.

Sendo assim, este caso demonstra que a hipótese de malignização de lesões diagnosticadas previamente como LPO pode se tratar de um diagnóstico inicial errôneo. Atualmente, os critérios para o diagnóstico de LPO já são bem definidos e são capazes de diferencia-lo de outras lesões. Vale ressaltar a importância do estomatologista e patologista oral no correto diagnóstico de lesões orais e de casos que exigem bastante precisão.

\section{CONCLUSÃO}

O LPO ainda é considerado pela OMS como uma doença potencialmente maligna devido a falta de unanimidade em relação ao risco de transformação maligna desta entidade patológica. É importante salientar que o diagnóstico de LPO requer a associação de características clínicas e microscópicas pré-determinadas, sendo sempre necessário o acompanhamento clínico desses casos. O erro de diagnóstico pode levar a morbidades importantes e comprometer o prognóstico do caso.

\section{REFERÊNCIAS}

1. Giuliani M, Troiano G, Cordaro M, Corsalini M, Gioco G, Lo Muzio L et al. Rate of malignant transformation of Oral Lichen Planus: a systematic review. Oral Dis. 2019; 25(3):693-709.

2. Bardellini E, Amadori F, Flocchini P, Bonadeo S, Majorana A. Clinicopathological features and malignant transformation of oral lichen planus: a 12-years retrospective study. Acta Odontologica Scandinavica 2013;71(3/4):834-40.

3. Eisen D. The clinical features, malignant potential, and systemic associations of oral lichen planus: a study of 723 patients. J Am Acad Dermatol. 2002;46(2):207-14.

4. Farhi D, Dupin N. Pathophysiology, etiologic factors, and clinical management of oral lichen planus, part I: facts and controversies. Clin Dermatol. 2010;28(1):100-8.

5. Roopashree MR, Gondhalekar RV, Shashikanth 
MC, George J, Thippeswamy SH, Shukla A. Pathogenesis of oral lichen planus - a review. J Oral Pathol Med. 2010;39(10):729-34.

6. van der Meij EH, van der Waal I. Lack of clinicopathologic correlation in the diagnosis of oral lichen planus based on the presently available diagnostic criteria and suggestions for modifications. J Oral Pathol Med. 2003;32(9):507-12.

7. Kramer IR, Lucas RB, Pindborg JJ, Sobin LH. Definition of leukoplakia and related lesions: an aid to studies on oral precancer. Oral Surg Oral Med Oral Pathol. 1978;46(4):518-39.

8. Aghbari SMH, Abushouk AI, Attia A, Elmaraezy A, Menshawy A, Ahmed MS et al. Malignant transformation of oral lichen planus and oral lichenoid lesions: a meta-analysis of 20095 patient data. Oral Oncology. 2017;68:92-102.

9. Eisenberg E. Oral lichen planus:a benign lesion. J Oral Maxillofac Surg. 2000;58(11):1278-85.

10.Alrashdan MS, Cirillo N, McCullough M. Oral lichen planus: a literature review and update. Arch Dermatol Res. 2016;308(8):539-51.

11.Lodi G, Scully C, Carrozzo M, Griffiths M, Sugerman PB, Thongprasom K. Current controversies in oral lichen planus: report of an international consensus meeting. Part 2. Clinical management and malignant transformation. Oral Surg Oral Med Oral Pathol Oral Radiol Endod. 2005;100(2):164-78.

12. Sousa FACG, Paradella TC, Brandão AAH, Rosa LEB. Líquen plano bucal versus displasia epitelial: dificuldades diagnósticas. Braz J Otorhinolaryngol. 2009;75(5):716-20.

13.Rode M, Kogoj-Rode M. Malignant potential of the reticular form of oral lichen planus over a 25year observation period in 55 patients from Slovenia. J Oral Sci. 2002;44(2):109-11.

14. Van der Meij EH, Mast H, Van der Waal I. The possible premalignant character of oral lichen planus and oral lichenoid lesions: a prospective five-year followup study of 192 patients. Oral Oncol. 2007;43(8):742-48.

15. Oliveira Alves MG, Almeida JD, Balducci I, Guimarães Cabral LA. Oral lichen planus: a retrospective study of 110 Brazilian patients. BMC Res Notes. 2010;3:157.

16. Radochová V, Dř́zhal I, Slezák R. A retrospective study of 171 patients with oral lichen planus in the East Bohemia-Czech Republic-single center experience. J Clin Exp Dent. 2014;6(5):e556-61.

17. Fitzpatrick S, Hirsch S, Gordon S. The malignant transformation of oral lichen planus and oral lichenoid lesions: a systematic review. J Am Dent Assoc. 2014;145(1):45-56.

18.Bermejo-Fenoll A, Sánchez-Siles M, LópezJornet P, Camacho-Alonso F, Salazar-Sánchez N.
A retrospective clinicopathological study of 550 patients with oral lichen planus in south-eastern Spain. J Oral Pathol Med. 2010;39(6):491-96.

19. Chaiyarit P, Ma N, Hiraku Y, Pinlaor S, Yongvanit P, Jintakanon D et al. Nitrative and oxidative DNA damage in oral lichen planus in relation to human oral carcinogenesis. Cancer Sci. 2005;96:553-59.

20.Georgakopoulou EA, Achtari MD, Achtaris M, Foukas PG, Kotsinas A. Oral lichen planus as a preneoplastic inflammatory model. J Biomed Biotechnol. 2012;2012:759626.

21. Accurso BT, Warner BM, Knobloch TJ, Weghorst CM, Shumway BS, Allen CM et al. Allelic imbalance in oral lichen planus and assessment of its classification as a premalignant condition. Oral Surg Oral Med Oral Pathol Oral Radiol Endod. 2011;112(3):359-66.

\section{CONFLITO DE INTERESSES}

Os autores declaram não haver conflitos de interesse.

\section{AUTOR PARA CORRESPONDENCIA}

\section{Ademar Takahama Júnior}

ademartjr@uel.br
Submetido em 08/10/2018

Aceito em 11/03/2019 